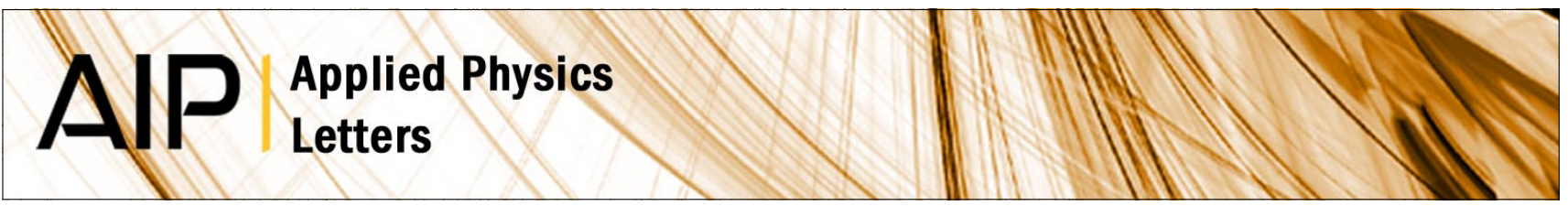

\title{
Improved electromechanical behavior in castable dielectric elastomer actuators
}

Samin Akbari, Samuel Rosset, and Herbert R. Shea

Citation: Appl. Phys. Lett. 102, 071906 (2013); doi: 10.1063/1.4793420

View online: http://dx.doi.org/10.1063/1.4793420

View Table of Contents: http://apl.aip.org/resource/1/APPLAB/v102/i7

Published by the American Institute of Physics.

\section{Related Articles}

Development of a non-explosive release actuator using shape memory alloy wire

Rev. Sci. Instrum. 84, 015005 (2013)

New Products

Rev. Sci. Instrum. 84, 019501 (2013)

Design and optimization of voice coil actuator for six degree of freedom active vibration isolation system using

Halbach magnet array

Rev. Sci. Instrum. 83, 105117 (2012)

The tracking control system of the VLT Survey Telescope

Rev. Sci. Instrum. 83, 094501 (2012)

Multi-functional dielectric elastomer artificial muscles for soft and smart machines

App. Phys. Rev. 2012, 7 (2012)

\section{Additional information on Appl. Phys. Lett.}

Journal Homepage: http://apl.aip.org/

Journal Information: http://apl.aip.org/about/about_the_journal

Top downloads: http://apl.aip.org/features/most_downloaded

Information for Authors: http://apl.aip.org/authors

\section{ADVERTISEMENT} \section{AIP $\begin{aligned} & \text { Applied Physics } \\ & \text { Letters }\end{aligned}$}

\section{EXPLORE WHAT'S NEW IN APL}

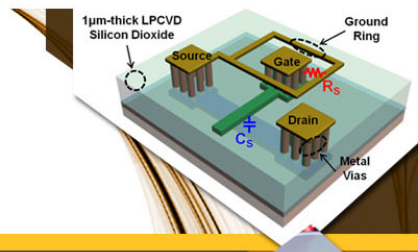

SURFACES AND INTERFACES

Focusing on physical, chemical, biological structural, optical, magnetic and electrical properties of surfaces and interfaces, and more.. 


\title{
Improved electromechanical behavior in castable dielectric elastomer actuators
}

\author{
Samin Akbari, ${ }^{\text {a) }}$ Samuel Rosset, and Herbert R. Shea \\ Microsystems for Space Technologies Laboratory, École Polytechnique Fédérale de Lausanne (EPFL), \\ Neuchâtel, Switzerland
}

(Received 14 December 2012; accepted 11 February 2013; published online 21 February 2013)

\begin{abstract}
Non-viscoelastic castable elastomers are replacing the polyacrylate VHB films in the new generations of dielectric elastomer actuators (DEAs) to achieve fast and reliable actuation. We introduce the optimum prestretch conditions to enhance the electromechanical behavior of the castable DEAs resulting in large actuation strain. For castable actuator in which the thickness is selected independent of the prestretch, uniaxial prestretch mode offers the highest actuation strain in the transverse direction compared to biaxial and pure shear. We experimentally demonstrate that miniaturization hinders the loss of tension and up to $85 \%$ linear actuation strain is generated with a $300 \times 300 \mu \mathrm{m}^{2}$ polydimethylsiloxanes-based DEA. (C) 2013 American Institute of Physics.

[http://dx.doi.org/10.1063/1.4793420]
\end{abstract}

Dielectric elastomer actuators (DEAs) are a fast growing class of electroactive polymer actuators. They combine large actuation strain and high energy density offering an appealing alternative to more conventional actuators. ${ }^{1-4}$ DEAs consist of a soft elastomeric membrane with compliant electrodes patterned on both sides. When a voltage is applied to the electrodes, the membrane is squeezed in thickness and expands in plane due to the electrostatic pressure. Giant voltagetriggered deformations up to $360 \%$ linear strain with clamped elastomer, ${ }^{5} 488 \%$ area strain with membrane under dead loads, ${ }^{6}$ and $1692 \%$ area strain on membranes mounted on an air chamber ${ }^{7}$ have been reported using polyacrylate VHB films from $3 \mathrm{M}$. However, to achieve reproducible and fast actuation and to prevent creep phenomenon, one must switch from VHB to materials with negligible viscoelastic behavior such as some classes of polydimethylsiloxanes (PDMS) or polyurethanes. Pelrine et al. have reported, in 2000, a $117 \%$ uniaxial linear strain with an actuator patterned as a long strip on a uniaxially prestretch silicone elastomer. ${ }^{1}$ More recently, Rosset et al. have shown a $60 \%$ linear planar strain on a PDMS based DEA with rise time of less than $10 \mathrm{~ms}^{8}$ However, the existing theoretical guidelines for large actuation deformation of DEAs developed based on VHB films cannot be directly implemented to castable elastomers. ${ }^{9,10}$ Unlike VHB, which is available as films with predefined thicknesses, PDMS or polyurethane are initially a resin and can be casted to form membranes of any desired thickness. This decouples the thickness of the elastomer from the prestretch ratio allowing to select them independently. For most practical materials, the thickness of the elastomer after prestretch is limited by design considerations and to avoid membrane rupture.

In this paper, we show that mechanism of large actuation strain for castable elastomers is different from the elastomeric films with predefined thickness, such as VHB. When the thickness reduction is excluded from the prestretch, the

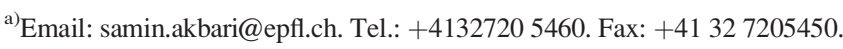

actuation voltage increases due to stiffening of the elastomer at high prestretch ratios. By theoretical analysis, we introduce the optimum prestretch ratio sufficient to suppress the pull-in instability allowing large deformation with the lowest actuation voltage. Compared to biaxial prestretch, uniaxial prestretch leads to higher voltage-induced strain in the transverse direction. However, the elastomer is more prone to lose tension in the direction with small prestretch ratio and fails by forming wrinkles. We experimentally demonstrate that miniaturization hinders the loss of tension and report up to $85 \%$ linear voltage-triggered strain with $300 \mu \mathrm{m} \times 300 \mu \mathrm{m}$ actuators on PDMS-based elastomers.

For the theoretical analysis, we consider an elastomeric membrane with initial dimensions of $\mathrm{L}_{1}, \mathrm{~L}_{2}$, and $\mathrm{t}_{0}$, which is then prestretched by ratios of $\lambda_{\mathrm{p} 1}$ and $\lambda_{\mathrm{p} 2}$ in directions 1 and 2 , respectively, to achieve the desired thickness $t_{i}$ for the device (Fig. 1). Constant forces, $\mathrm{P}_{1}$ and $\mathrm{P}_{2}$, are generated by a frame corresponding to the prestretch ratios and the strain energy of the elastomer. Then, the compliant electrodes assumed to cause no stiffening for this analysis are patterned over the whole surface and a voltage is applied to the electrodes reducing the thickness due to the Maxwell stress, which results in planar expansion. Since the material is incompressible, $\lambda_{1} \lambda_{2} \lambda_{3}=1$, where $\lambda_{\mathrm{i}}$ is the stretch ratio in direction $\mathrm{i}$.

The electrostatic pressure on the elastomer due to the applied voltage and the mechanical stress by the external forces on the elastomer deform the membrane till they balance with the Cauchy stress in the elastomer. The governing equations of the DEA are as follows: ${ }^{9-11}$

$$
\begin{aligned}
\sigma_{11}\left(\lambda_{1}, \lambda_{2}, \lambda_{3}\right) & =\lambda_{1} \frac{\partial \mathrm{W}\left(\lambda_{1}, \lambda_{2}, \lambda_{3}\right)}{\partial \lambda_{1}}-\lambda_{3} \frac{\partial \mathrm{W}\left(\lambda_{1}, \lambda_{2}, \lambda_{3}\right)}{\partial \lambda_{3}} \\
& =\varepsilon \mathrm{E}^{2}+\sigma_{p 1}, \\
\sigma_{22}\left(\lambda_{1}, \lambda_{2}, \lambda_{3}\right) & =\lambda_{2} \frac{\partial \mathrm{W}\left(\lambda_{1}, \lambda_{2}, \lambda_{3}\right)}{\partial \lambda_{2}}-\lambda_{3} \frac{\partial \mathrm{W}\left(\lambda_{1}, \lambda_{2}, \lambda_{3}\right)}{\partial \lambda_{3}} \\
& =\varepsilon \mathrm{E}^{2}+\sigma_{p 2}
\end{aligned}
$$




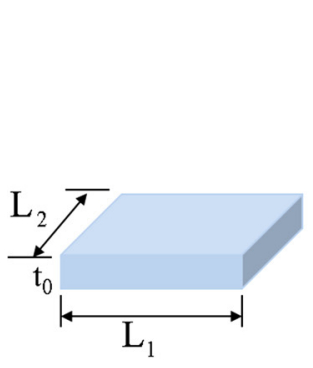

a)

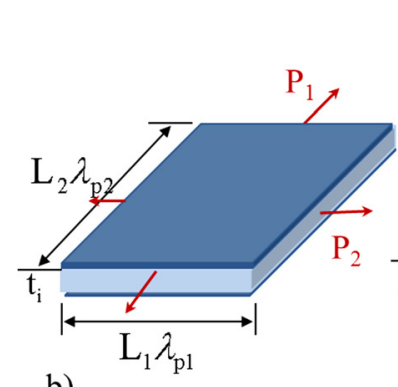

b)

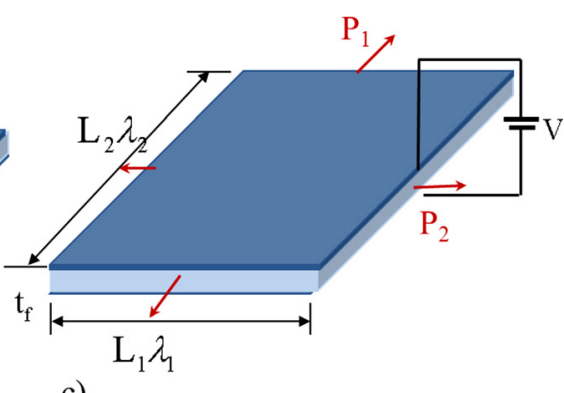

c)

FIG. 1. Schematic of a dielectric elastomer actuator: (a) A membrane is of initial dimensions of $\mathrm{L}_{1}, \mathrm{~L}_{2}$, and $\mathrm{t}_{0}$. (b) The membrane is prestretched by ratios of $\lambda_{\mathrm{p} 1}$ and $\lambda_{\mathrm{p} 2}$ in directions 1 and 2 , respectively and is subjected to constant forces corresponding to the prestretch ratios. (c) Compliant electrodes are patterned and a voltage is applied to the electrodes leading to planar expansion and thickness reduction.

where $\sigma_{11}$ and $\sigma_{22}$ are the principle Cauchy stresses of the elastomer in directions 1 and 2, respectively. $\sigma_{\mathrm{p} 1}$ and $\sigma_{\mathrm{p} 2}$ are the mechanical stresses due to the external forces, which are equal to the Cauchy stresses at the prestretched state with zero voltage. $\mathrm{W}\left(\lambda_{1}, \lambda_{2}, \lambda_{3}\right)$ is the strain energy density function of the elastomer derived by fitting the Gent hyperelastic material model to the experimental stress-strain curve obtained from the uniaxial test by a pull test instrument (Instron ${ }^{\circledR} 3343$, Noorwood, USA). Gent strain energy function models the strain stiffening of the elastomers and is defined as ${ }^{12}$

$$
\mathrm{W}=\frac{-\mu \mathrm{J}_{\mathrm{m}}}{2} \ln \left(1-\frac{\lambda_{1}^{2}+\lambda_{2}^{2}+\lambda_{3}^{2}-3}{\mathrm{~J}_{\mathrm{m}}}\right),
$$

where $\mu$ is the shear modulus and $\mathrm{J}_{\mathrm{m}}$ is the second material constant related to the limiting stretch. When $\lambda_{1}{ }^{2}+\lambda_{2}{ }^{2}$ $+\lambda_{3}{ }^{2}-3$ approaches to $J_{\mathrm{m}}$, the Gent model stiffens sharply. For Sylgard 186, which has short polymer chains, $\mu$ and $\mathrm{J}_{\mathrm{m}}$ are measured $0.59 \mathrm{MPa}$ and 30.04 , respectively.

Equations (1) and (2) are combined and $\lambda_{3}$ is substituted with $1 / \lambda_{1} \lambda_{2}$ after differentiation. The prestretch in the elastomer is simulated as two constant forces calculated from the Cauchy stresses at the stretch state of $\lambda_{\mathrm{p} 1}$, and $\lambda_{\mathrm{p} 2}$ and zero electric field (Fig. 1(b)). The governing equations become as following:

$$
\begin{aligned}
\varepsilon \lambda_{1}{ }^{2} \lambda_{2}{ }^{2} \frac{\mathrm{V}^{2}}{t_{0}{ }^{2}}= & \frac{\mu\left(\lambda_{1}{ }^{2}-\lambda_{1}{ }^{-2} \lambda_{2}{ }^{-2}\right)}{1-\left(\lambda_{1}{ }^{2}+\lambda_{2}{ }^{2}+\lambda_{1}{ }^{-2} \lambda_{2}{ }^{-2}-3\right) / \mathrm{J}_{\mathrm{m}}} \\
& -\sigma_{11}\left(\lambda_{p 1}, \lambda_{p 2}\right) \cdot \frac{\lambda_{1}}{\lambda_{p 1}}, \\
\varepsilon \lambda_{1}{ }^{2} \lambda_{2}{ }^{2} \frac{\mathrm{V}^{2}}{t_{0}^{2}}= & \frac{\mu\left(\lambda_{2}^{2}-\lambda_{1}{ }^{-2} \lambda_{2}{ }^{-2}\right)}{1-\left(\lambda_{1}{ }^{2}+\lambda_{2}{ }^{2}+\lambda_{1}{ }^{-2} \lambda_{2}{ }^{-2}-3\right) / \mathrm{J}_{\mathrm{m}}} \\
& -\sigma_{22}\left(\lambda_{p 1}, \lambda_{p 2}\right) \cdot \frac{\lambda_{2}}{\lambda_{p 2}} .
\end{aligned}
$$

When a $50 \mu \mathrm{m}$ thick membrane is biaxially prestretched at different ratios and subjected to a high voltage, the electromechanical response is enhanced as shown in Fig. 2(a). The pull-in instability is hindered due to the prestretch force and the actuation voltage is reduced as a consequence of thickness reduction by prestretching. This type of electromechanical response has been reported in several articles based on theoretical or experimental results, in which VHB films were used in making the DEAs. ${ }^{5,9-11,13,14}$ When a constant thickness after prestretching $(30 \mu \mathrm{m})$ is considered for the actuator, increasing the biaxial prestretch ratio leads to higher actuation voltage, as shown in Fig. 2(b). Biaxial prestretch of 1.5 is sufficient to suppress the pull-in instability and further prestretching deteriorates the actuator's performance by stiffening it and increasing the required actuation voltage for a given displacement.

Uniaxial prestretching offers a more appealing enhancement in DEAs performance in the case of fixed thickness after prestretch. The elastomer stiffens in the prestretched direction leading to higher actuation strain in the transverse direction. The pull-in instability is also suppressed but at higher prestretch ratios compared to the biaxial prestretch. The minimum uniaxial prestretch ratio to achieve monotonic voltage-strain curve is 2.75 for Sylgard 186, as shown in Fig. 3 . The actuation stretch along and perpendicular to the prestretch direction respect to the applied voltage is plotted in Figs. 3(a) and 3(b), respectively. By increasing the prestretch ratio, the voltage-triggered stretch reduces along the prestretched direction and increases in the transverse direction. In contrast with the biaxial prestretch, the actuation voltage is not increased with the prestretch ratio and therefore, much higher strain can be achieved in the transverse direction
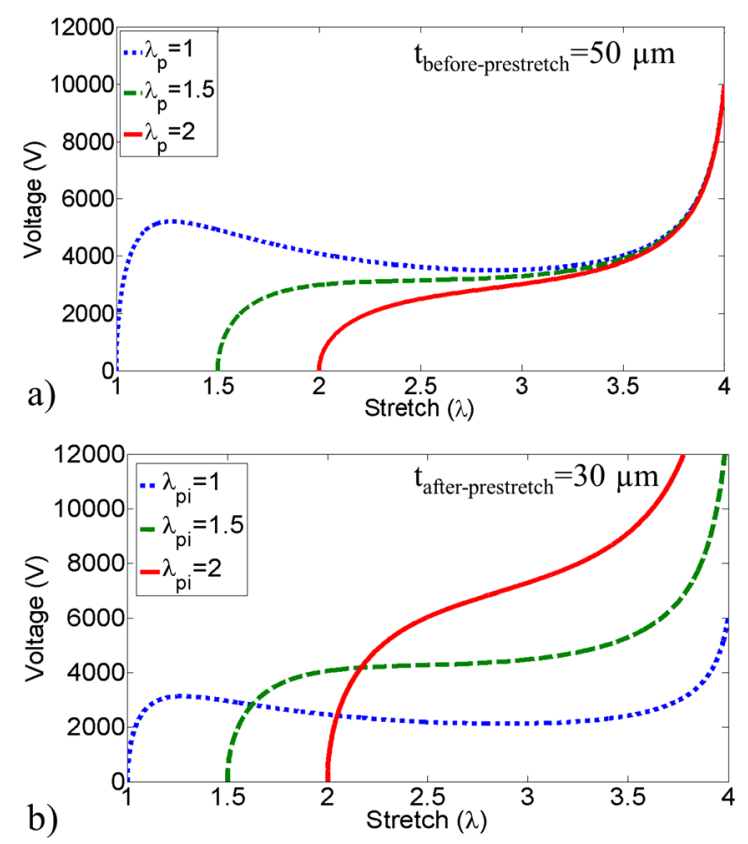

FIG. 2. (a) Electromechanical behavior of a $50 \mu \mathrm{m}$ thick elastomer (Sylgard 186) at different biaxial prestretch ratios. The pull-in instability is suppressed by prestretching and the actuation voltage is reduced due to thickness reduction because of prestretching. (b) Thickness of the actuator after biaxial prestretching is fixed to $30 \mu \mathrm{m}$. A prestretch ratio of 1.5 is sufficient to suppress the pull-in instability and a higher prestretch ratio is not desirable as it stiffens the elastomer and deteriorates the performance of the actuator. 

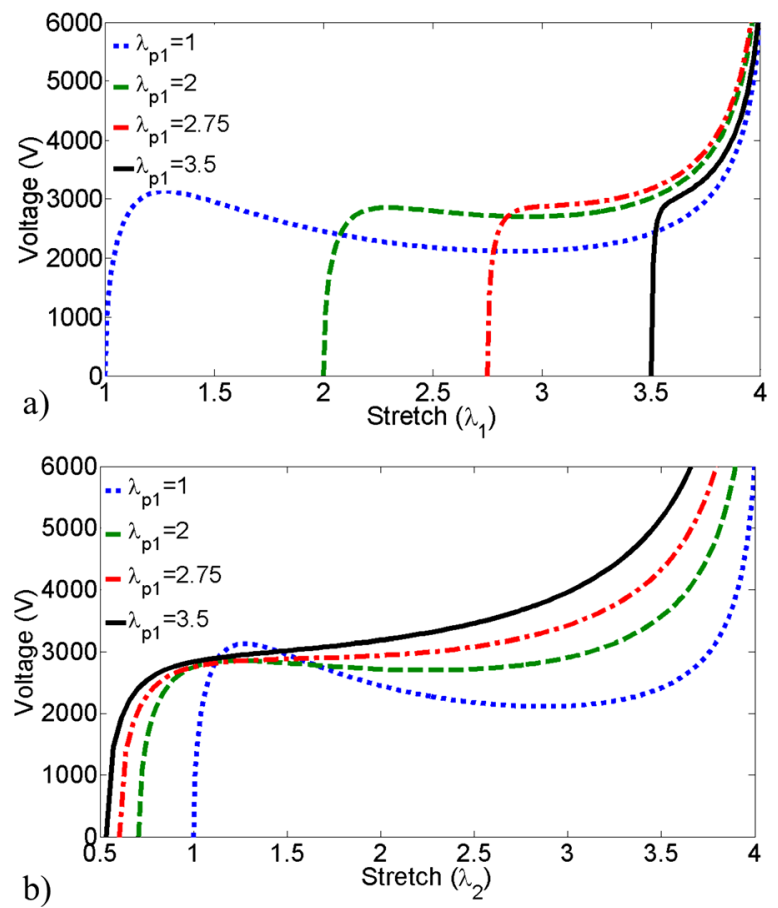

FIG. 3. Electromechanical behavior of a dielectric elastomer actuator on uniaxially prestretched membranes. (a) Along the prestretched direction. (b) Perpendicular to the prestretched direction. Higher voltage-triggered stretch can be achieved in the transverse direction without increasing the actuation voltage. In the theoretical calculations, the thickness of elastomer is fixed to $30 \mu \mathrm{m}$ after prestretch.

before electrical break down of the elastomer. It should be noted that uniaxial prestretching leads to anisotropic strain profile approaching to uniaxial at high prestretch ratios.

Prestretching the elastomer is a key factor for enhancing the electromechanical response of dielectric elastomer actuators as it suppresses the pull-in instability and allows higher actuation strain. However, there is an optimum prestretch ratio sufficient to suppress the pull-in instability for each type of elastomer and prestretch mode. Higher prestretch ratios will unnecessarily stiffen the elastomer and increase the actuation voltage. The voltage-triggered stretch $\left(\lambda_{2} / \lambda_{\mathrm{P} 2}\right)$ of the elastomer at the optimum ratios under different prestretch modes (biaxial, pure shear, uniaxial) is plotted in Fig. 4. Above the optimum ratios, there is no instability and the Hessian of the free energy function is definite positive, as first introduced by Zhao and Suo ${ }^{15}$ For Sylgard 186, that has short polymer chains and elongation at break of 4 , a biaxial prestretch of 1.5 is enough to achieve monotonic voltagestrain curve. Higher prestretch ratios are required for pure shear $\left(\lambda_{\mathrm{p} 2}=1\right)$ and uniaxial prestretch modes. The maximum response is then limited by breakdown voltage of the elastomer. The uniaxial prestretch mode allows the highest actuation stretch (2.38) in the transverse direction, where the membrane is the softest. It should be noted that the theoretical actuation strains are calculated by assuming free boundary conditions. The compressive stress by the passive membrane when the electrodes are patterned on the center of a membrane fixed on a frame is neglected.

To verify our theoretical predictions, we have tested different actuators at multiple prestretch modes. Silicone

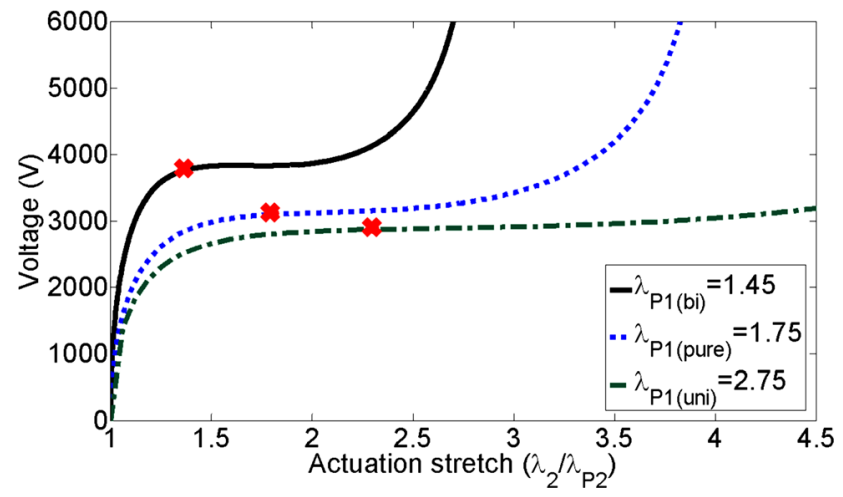

FIG. 4. Theoretical prediction of voltage-induced stretch defined as $\lambda / \lambda_{\mathrm{P}}$ is plotted at the optimum prestretch ratios for biaxial, pure shear, and uniaxial prestretch modes. Further prestretching ratios will redundantly stiffen the elastomer. The red crosses indicate the breakdown electric field of $250 \mathrm{~V} / \mu \mathrm{m}$. The highest actuation strain is predicted in the transverse direction of a uniaxially prestretched membrane, where the membrane is the softest. The calculated ratios are for a silicone elastomer, Sylgard 186, $30 \mu \mathrm{m}$ thick after prestretch.

membranes (Sylgard 186) with various thicknesses are casted using an applicator and prestretched with different prestretch ratios to achieve $30 \mu \mathrm{m}$ thick elastomer and then fixed on a frame. ${ }^{16}$ Carbon powder is dispersed in an elastomeric matrix and stamped at the center of the frame to pattern thin compliant electrodes ${ }^{17}$ on both sides of the membrane. Then, the inplane voltage-induced stretches of the actuators are recorded and compared with the theoretical calculations, as shown in Fig. 5. We have observed that the electric field strength of the elastomer is enhanced from $100 \mathrm{~V} / \mu \mathrm{m}$ for nonstretched films $\mathrm{s}^{4,16,18}$ to $250 \mathrm{~V} / \mu \mathrm{m}$ by 1.75 biaxial prestretch.

Since a thin membrane cannot sustain compressive strain, the actuators are prone to lose tension in the direction with a small prestretch ratio in the anisotropic prestretch mode. As the actuated area expands due to the voltage, it experiences compressive stress from the passive area till the tensile stress on the actuator becomes zero and the membrane buckles out of plane in the form of wrinkles, ${ }^{19}$ as shown in Figs. 6(a) and 6(b). The actuator is $1.5 \mathrm{~mm}$ diameter patterned on a $20 \mathrm{~mm}$ diameter membrane fixed on a frame. The prestretch ratios are 2.75 and 0.9 in the $y$ and $x$ directions, respectively. The

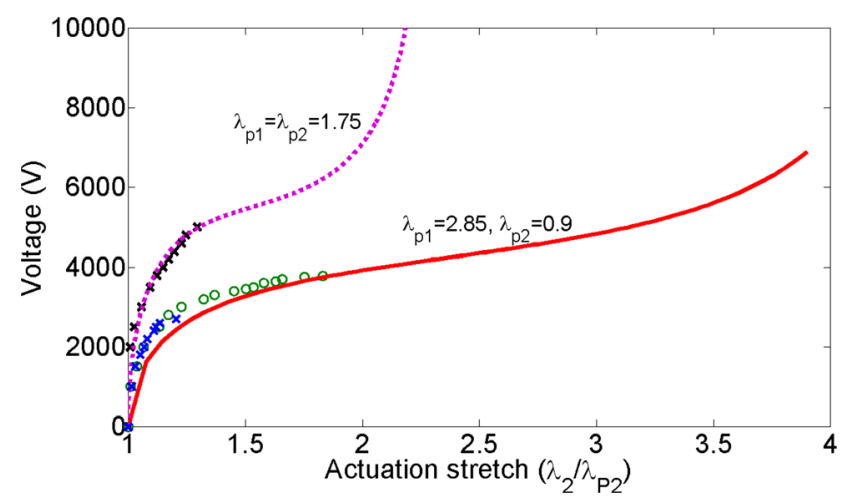

FIG. 5. Voltage-induced stretch of dielectric elastomer actuators made with two different prestretch conditions. The $\mathrm{x}$ and o data points are the measured linear stretch expansion on $1.5 \mathrm{~mm}$ diameter and $300 \times 300 \mu \mathrm{m}^{2}$ actuators, respectively. The actuator under biaxial prestretch is failed at 1.32 stretch (32\% strain) due to electric breakdown at $250 \mathrm{~V} / \mu \mathrm{m}$. The actuators with anisotropic prestretch mode failed due to the loss of tension at $20 \%$ and $85 \%$ strain for $1.5 \mathrm{~mm}$ diamter and $300 \times 300 \mu \mathrm{m}^{2}$ actuators, respectively. 

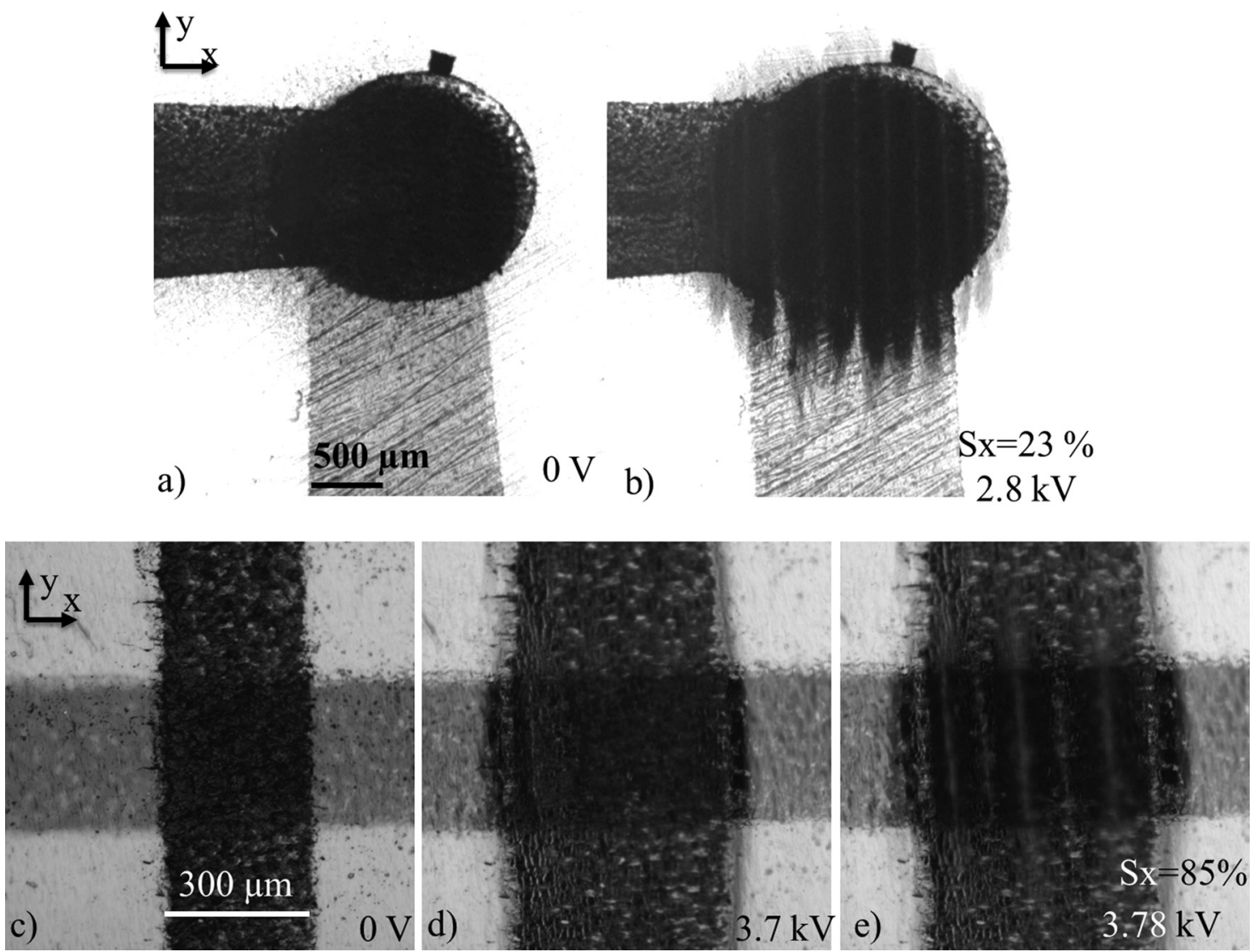

FIG. 6. (a) $1.5 \mathrm{~mm}$ diameter DEA is made on a membrane with prestretch ratios of $\lambda_{\mathrm{px}}=0.9, \lambda_{\mathrm{py}}=2.6$ and fixed on a $20 \mathrm{~mm}$ frame. (b) The actuator experiences loss of tension at $23 \%$ linear strain and wrinkles appear on the membrane. (c) $300 \mu \mathrm{m} \times 300 \mu \mathrm{m}$ DEA is made on a on a $31 \mu \mathrm{m}$ thick silicone elastomer with prestretch ratios of $\lambda_{\mathrm{px}}=0.9, \lambda_{\mathrm{py}}=2.8$. (d) The actuator generates $70 \%$ actuation strain in $\mathrm{x}$ direction at $3.7 \mathrm{kV}$. (e) The actuator experiences loss of tension at $85 \%$ linear strain $(1.85$ stretch $)$ at $3.78 \mathrm{kV}$.

loss of tension occurs at $20 \%$ strain (1.2 actuation stretch) and prohibits higher actuation stretch predicted by the theoretical analysis, in which the electromechanical equations were solved under free boundary conditions.

Miniaturization of the actuators effectively holds back the loss of tension allowing higher actuation strain. We fabricated $300 \mu \mathrm{m} \times 300 \mu \mathrm{m}$ actuators on a $31 \mu \mathrm{m}$ thick elastomer with prestretch ratios of $\lambda_{\mathrm{py}}=2.8$ and $\lambda_{\mathrm{px}}=0.9$, which is fixed on a $40 \mathrm{~mm}$ diameter frame. The actuation strain enhanced by 4 times compared to the $1.5 \mathrm{~mm}$ diameter actuator and $85 \%$ linear strain (1.85 stretch) achieved till the wrinkles appeared, as shown in Figs. 6(c)-6(e).

In a simplified model, when the linear stretches are matched between the passive and active regions at the equilibrium, we have

$$
\lambda_{1 \mathrm{pas}}=1-\frac{L_{1 \mathrm{act}}}{L_{1 \mathrm{pas}}}\left(\lambda_{1 \mathrm{act}}-1\right)
$$

where $\mathrm{L}_{1 \text { act }}$ and $\mathrm{L}_{1 \text { pas }}$ are the initial lengths, and $\lambda_{\text {1act }}$ and $\lambda_{1 \text { pas }}$ are the linear stretch ratios of the active and passive regions in direction 1 . Decreasing the size of the active to passive area reduces the stretch ratio and the consequent Cauchy stress in the passive region. Therefore, the effect of the passive region on the actuator diminishes by scaling down the actuator, holding back the loss of tension. The same effect can be achieved by enlarging the passive area. To precisely analyze effect of the passive region, its inhomogeneous stress field should be analyzed considering a continuous deformation between the passive and active region as demonstrated by Koh et al. ${ }^{9}$ and Plante and Dubowsky. ${ }^{14}$

In conclusion, we demonstrated that the existing theoretical guidelines for large actuation strains based on VHB films cannot be directly implemented for castable elastomers. Since they can be casted at any thickness, it is more effective to select a thinner membrane rather than highly prestretching it for the actuator. We theoretically calculated the optimum prestretch ratio, which is sufficient to suppress the elastomer and above which the elastomer gets redundantly stiff increasing the actuation voltage. We experimentally verified the theoretical predictions and demonstrated that miniaturization hinders the loss of tension failure mode allowing higher actuation strain.

This work was funded by Swiss National Foundation grant \#200020-140394. The authors thank Mr. Luc Maffli for helpful discussions.

${ }^{1}$ R. Pelrine, R. Kornbluh, Q. Pei, and J. Joseph, Science 287(5454), 836 (2000).

${ }^{2}$ P. Brochu and Q. Pei, Macromol. Rapid Commun. 31(1), 10 (2010).

${ }^{3}$ G. Kovacs, P. Lochmatter, and M. Wissler, Smart Mater. Struct. 16(2), S306 (2007).

${ }^{4}$ S. Akbari and H. R. Shea, J. Micromech. Microeng. 22(4), 045020 (2012).

${ }^{5}$ M. Kollosche, J. Zhu, Z. Suo, and G. Kofod, Phys. Rev. E 85(5), 051801 (2012). 
${ }^{6}$ J. Huang, T. Li, C. Chiang Foo, J. Zhu, D. R. Clarke, and Z. Suo, Appl. Phys. Lett. 100(4), 041911 (2012).

${ }^{7}$ C. Keplinger, T. Li, R. Baumgartner, Z. Suo, and S. Bauer, Soft Matter 8(2), 285 (2012).

${ }^{8}$ S. Rosset, P. Gebbers, B. M. O'Brien, and H. R. Shea, Proc. SPIE 8340, 83400412 (2012).

${ }^{9}$ S. J. A. Koh, T. Li, J. Zhou, X. Zhao, W. Hong, J. Zhu, and Z. Suo, J. Polym. Sci., Part B: Polym. Phys. 49(7), 504 (2011).

${ }^{10}$ X. Zhao and Z. Suo, Phys. Rev. Lett. 104(17), 178302 (2010).

${ }^{11}$ Z. Suo, Acta Mech. Solida Sinica 23(6), 549-578 (2010). Available at http://www.sciencedirect.com/science/article/pii/S0894916611600049.
${ }^{12}$ A. N. Gent, Rubber Chem. Technol. 69(1), 59 (1996).

${ }^{13}$ T. Lu, J. Huang, C. Jordi, G. Kovacs, R. Huang, D. R. Clarke, and Z. Suo, Soft Matter 8(22), 6167 (2012).

${ }^{14}$ J.-S. Plante and S. Dubowsky, Int. J. Solids Struct. 43(25-26), 7727 (2006).

${ }^{15}$ X. Zhao and Z. Suo, Appl. Phys. Lett. 91(6), 061921 (2007).

${ }^{16}$ S. Akbari and H. R. Shea, Sens. Actuators, A 186(0), 236 (2012).

${ }^{17}$ S. Rosset and H. R. Shea, Appl. Phys. A 110(2), 281 (2013).

${ }^{18}$ S. Rosset, M. Niklaus, P. Dubois, and H. R. Shea, J. Microelectromech. Syst. 18(6), 1300 (2009).

${ }^{19}$ A. T. Conn and J. Rossiter, Appl. Phys. Lett. 101(17), 171906 (2012). 